\title{
Chronological study in microanatomy of human foetal pancreatic tissue
}

\author{
T.V. Ramani ${ }^{1, *}$, M. Pratyusha ${ }^{2}$, S. Saritha ${ }^{3}$ \\ ${ }^{\mathbf{1}}$ Assistant Professor, ${ }^{\mathbf{2}}$ M.B.B.S Student, ${ }^{3}$ Professor and HOD, Dept. of Anatomy, ${ }^{\mathbf{1}, \mathbf{3}}$ Kamineni Academy of Medical Sciences and \\ Research Centre, Hyderabad, ${ }^{2}$ Shadan Institute of Medical Sciences, Hyderabad, Telangana, India
}

\section{*Corresponding Author: T.V. Ramani}

Email: ramani_muddaloor@hotmail.co.uk

Received: $13^{\text {th }}$ May, 2018

Accepted: $24^{\text {th }}$ July, 2018

\begin{abstract}
Introduction: The name of pancreas derives from the Greek' word. The pancreas involves in diseases like diabetes mellitus and pancreatic cancer. It is contains Endocrine cells or Islets of Langerhans that secrete insulin, glucagon and somatostatin, which regulate blood glucose levels. ${ }^{1}$

The exocrine pancreatic acini secretes pancreatic enzymes and alpha fetoprotein which change the expression level during development and influences development and differentiation of pancreas. ${ }^{2}$

Endocrine disease causes diabetes which affects at least 200 million people worldwide, and this number is to be doubled by $2025 .{ }^{1}$ The exocrine part leads to pancreatic cancer causing about 65,000 deaths in European countries which is virtually incurable.

Aims: The purpose of the current study was to investigate microanatomy (Histogenesis) of Pancreas in aborted fresh human foetuses without any congenital abnormality. So the present study was conducted to know the development of acini and islets and their clinical correlation.

Materials and Methods: Foetuses aged between 12 to 40 weeks of gestational age and they were collected from Obstetrics and Gynaecology department of Kamineni Institute of Medical Sciences, Narkatpally, Nalgonda (Dt), Telangana, (India) and Kamineni Academy of Medical Sciences and Research centre, L.B. Nagar, Hyderabad.

The histogenic procedures were employed at all gestational ages and were discussed with the available literature because various studies have proved that the islets of pancreas are necessary for the transplantation of pancreas, but very few studies showed the importance of pancreatic acini.

Results and Conclusion: In the early weeks (12-18 weeks) the parenchyma forms primitive tubules with small lobes and lobules of acini and small spherical islets. As the age advances (18-24 weeks) the size and number of Islets are increased with encapsulation and proliferation of acinar cells.

To conclude, at the age of 18-24 weeks the fetal pancreas is suitable for organ transplantation in patients with diabetes mellitus and pancreatic cancer.
\end{abstract}

Keywords: Histogenesis, Gestational period, Pancreas, Serous acini, Islets of Langerhans, Microanatomy, Ducts, Alpha fetoprotein (AFP).

\section{Introduction}

Pancreas is a exocrine and endocrine organ, a retort flask shaped, present in the epigastric and left hypochondric regions. It has two ducts, main duct and an accessory pancreatic duct. ${ }^{3}$

It is developed by two endo dermal buds, dorsal and ventral, which later fuse and forms pancreas at around $7^{\text {th }}$ week Intra Uterine Life.

Main and accessory pancreatic ducts starts developing around $6^{\text {th }}$ week of intra uterine period. Development of Pancreatic acini and Islets of Langerhans starts from $12^{\text {th }}$ week of intra uterine life. ${ }^{3,4}$ Islets of Langerhans are proportionately more numerous in foetuses and infants than in older individuals. Paul Langerhans Jr. discovered Islets of Langerhans in 1869 and insulin by Banting and Best in 1921. ${ }^{5}$

The exocrine acini derived from the repeated sprouting of the ducts at 12 weeks of IUL. These are arranged in the form of lobes and lobules with thin intra lobular septa which contain blood vessels, nerves and ducts.
The acinar cells or some times duct system give rise to the endocrine part of islets of langerhans. These are later get detatched and forms independent colonies. These islets are scattered throughout the parenchyma and are more in the tail of the pancreas. ${ }^{6,7}$

In the fetus, the Islets are arranged in single or in small groups with a structural organisation of four cell types which has functional significance. The beta cell occupy the central core of an Islet and other cells Alpha, Delta, PP cells are located at the peripheral region. ${ }^{8}$

Many studies have proved that transition from Islets to acini or from acini to Islets may occur and both are not independent structures developmentally. ${ }^{5}$

Alpha foeto protein which is the most abundant serum protein in foetus produced by liver and later by pancreatic acini during embryogenesis, but is transiently seen in fetal pancreatic acini during development. It was first identified in the serum of human foetuses by Bergstand and Czar in 1956. ${ }^{9}$

Islets are difficult to isolate from adult human pancreas therefore the aim of the present light 
microscopic study in order to describe the development of the human fetal pancreas and to distinguish histological differentiation of exocrine and endocrine components of pancreas by employing haematoxylin and eosin techniques, because this is in relevance with fetal pancreatic tissue transplants in diabetes mellitus type 1 and in pancreatic cancer.

\section{Materials and Methods}

Materials: Human foetuses of 12 to 40 weeks (50) were obtained from Obstestric and gynaecology department of Kamineni Institute of Medical sciences and Kamineni academy of medical sciences and research centre. After obtaining ethical committee approval and these Foetuses were arranged in 5 groups of 6 weeks interval.

Pancreas was dissected and sent to the histological preparation for staining. These were studied under the microscope.

The recent study of the literature showed 3 stages of development of fetal Islets of pancreas ${ }^{10}$

(a) Single cells (b) Inselfield (c) Mantellinsel

\begin{tabular}{|l|c|}
\hline Grouping of foetuses in weeks \\
\hline Group 1 & 12 to 18 \\
\hline Group 2 & 18 to 24 \\
\hline Group 3 & 24 to 30 \\
\hline Group 4 & 30 to 36 \\
\hline Group 5 & 36 to term \\
\hline
\end{tabular}

\section{Observations and Results}

Observations of 12 to 18 weeks Fetus: Fig. 1a \&1b

1. Branched tubules of mesenchymal tissue was seen with wide lumen.

2. At the end of the tubules budding was seen, with formation of primitive acini.

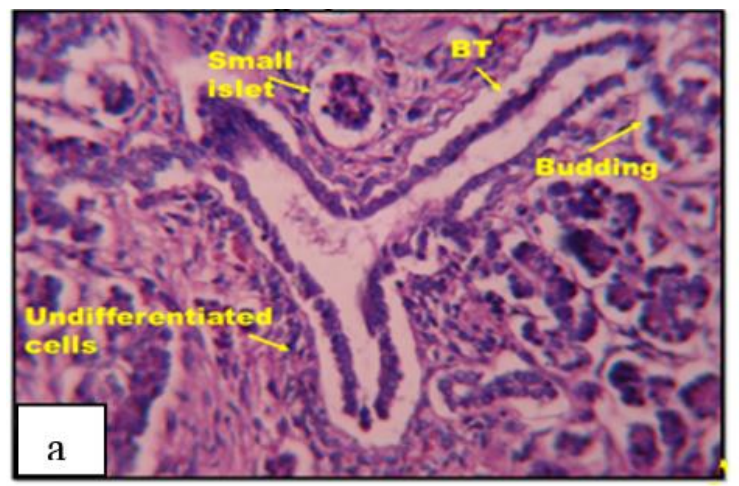

3. Throughout the mesenchyme undifferentiated cells were seen.

4. From terminal process of duct system Small, single islet cells having an ill-defined capsule without capillaries were seen. (Single cell stage).

5. Lobes and lobules of parenchyma was about to be seen.

Observations of 18 to 24 weeks Fetus: Fig. 2a \&2b

1. Mesenchymal tissue is reduced with proliferation of acinar cells

2. Small lobes and lobules were established.

3. Intra lobular ducts were seen and are lined by cuboidal cells.

4. The islet size is increased, arranged in small groups and entrapped within the acinar cells. (Inselfield stage)

Observations of 24 to 30 weeks Fetus: Fig. 3

1. Well-formed lobes and lobules of acini were seen.

2. Undifferentiated cells (Islet of Langerhans) as clumps, from the intra lobular duct was also observed. (Mantel field stage)

Observations of 30 to 36 weeks Fetus: Fig. 4

1. Groups of Islets of Langerhans are well differentiated, with a thin capsule and capillaries.

2. Lobes and lobules are well formed with packed serous acini.

3. Duct system was well developed within the thick Interlobular septa.

Observations of 36 to Full term: Fig. 5 a \& $\mathbf{5 b}$

1. The adult format of the microscopic structure of pancreas was seen.

2. Lobes and lobules of mature acini surrounding a large, vascular islets with thin inter lobar septa were seen in all term foetuses (stage 3 Mantellinsel).

No further developmental changes were seen.

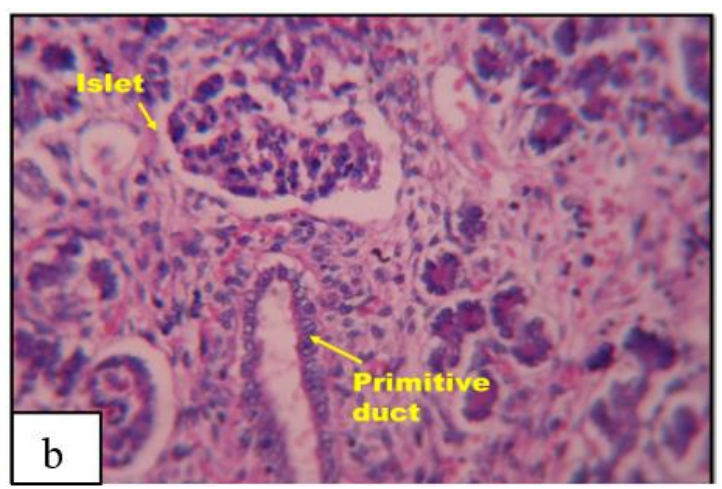

Fig. 1a: Group 1 (12-18 weeks) Photomicrograph 400x H\&E; 1b: Group 1 (12-18 weeks) Photomicrograph 400 X H\&E 

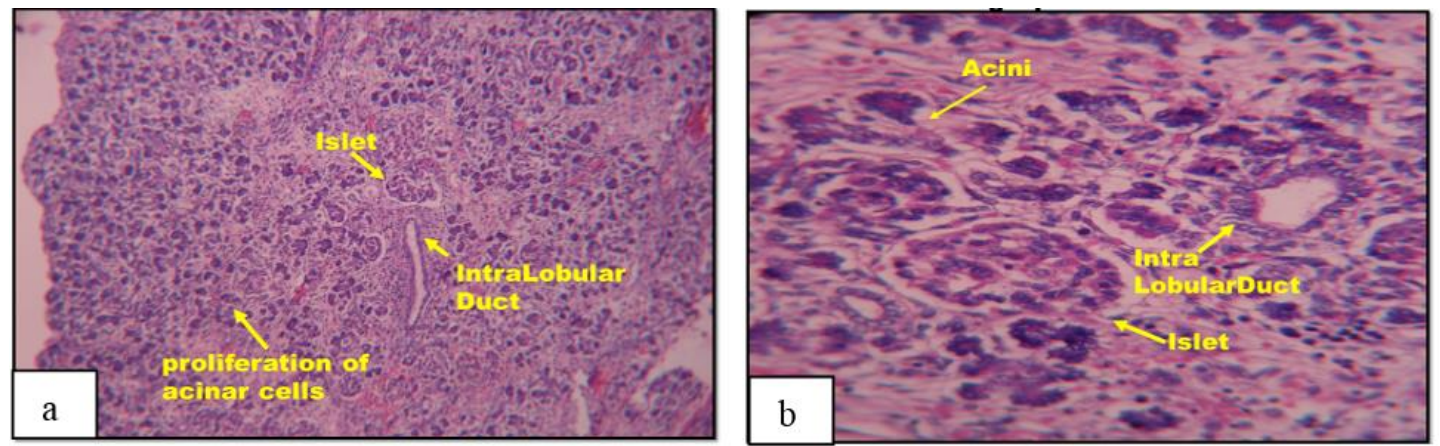

Fig. 2a: Group 2 (18-24 weeks) photomicrograph 100 X H\&E; 2b: Group 2 (18-24 weeks) photomicrograph 400 X H\&E

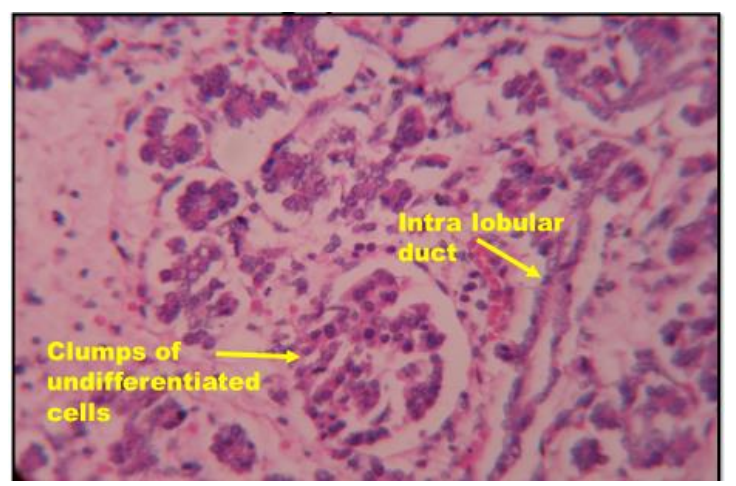

Fig. 3: Group 3 (18-24 weeks) photomicrograph 400 X H\&E

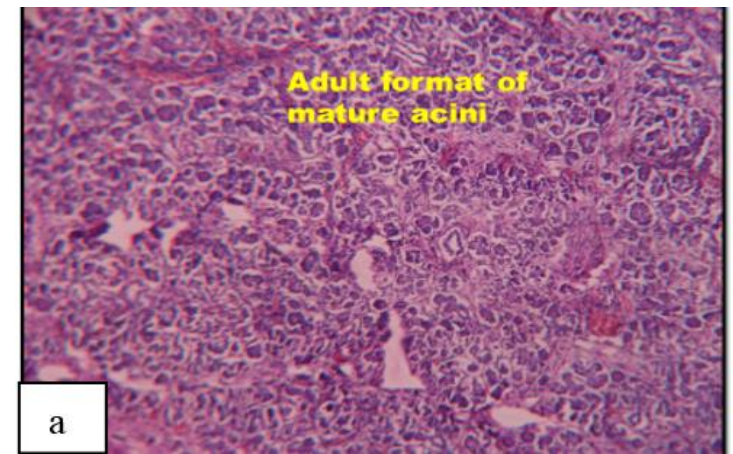

Fig. 5a: Group 5 (full term) photomicrograph $100 \times$ H\&E; 5b: Group 5 (full term) photomicrograph 400 X H\&E

\section{Discussion \\ Development includes three Fundamental Processes: Growth, differentiation and metabolism. Growth is increase in spatial dimensions and in weight. Differentiation is increase in complexity and organisation. This differentiation may not be apparent at first, but when apparent it is known as 'Histogenesis'. ${ }^{11}$ \\ Mammals, birds, reptiles and amphibians have a pancreas with similar histological features of development. While in some fish, the islet cells are segregated as Brockmann bodies. Invertebrates do not have a pancreas, but comparable endocrine cells may be found in the gut or in the brain. ${ }^{2}$}

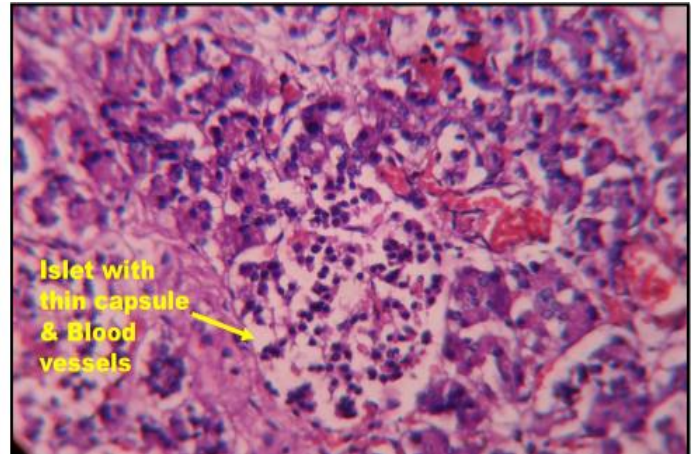

Fig. 4: Group 4 (30-36 weeks) photomicrograph 400 $\mathrm{X} \mathbf{H} \& \mathrm{E}$

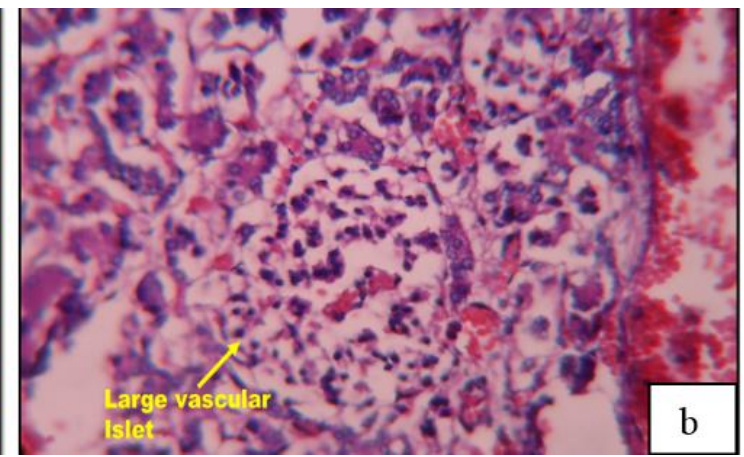

The diabetes mellitus occurs following total pancreatectomy in the dog was discovered by Mering and Minkowski which marked the beginning of 'pancreatic era'. ${ }^{12}$

Pancreatic extract was prepared by Banting and Best in 1922 and was used for the treatment of diabetes mellitus. Recently clinicians are making an attempt to treat the patients of insulin dependent diabetes mellitus by transplantation of foetal pancreas for the successful cell replacement therapies. ${ }^{12}$

The initial study of pancreatic islet formation in the human foetus was reported by Pearce (1903).

The alpha fetoprotein a major serum protein during development is one of the earliest protein to be synthesised by the embryonic liver. It is subsequently expressed in the developing exocrine part of pancreas. 
AFP expression in Human embryos can catabolise amino acids which provide an important supply of metabolic energy for the embryo. ${ }^{9}$ AFP is used as a serological marker for germ cell tumors and screening of neural tube defects and Down's syndrome prenatally (Abelev \& Eraiser, 1999) because the synthesis of AFP decreases dramatically after birth.

In the present work light microscopic examination of pancreatic section was divided in 5 groups. The first group was of 12-18 weeks of gestation, second group 18-24 weeks, group 324 to 30 weeks, group 430 to 36 weeks and finally group 5 includes full term pancreatic architecture.

In the early weeks of maturation, parenchyma was composed of small lobes and lobules with minimum amount of mesenchymal tissue. Undifferentiated mesenchymal tissue was seen up to the 40 weeks of age. As maturity increased, mesenchymal tissue decreased due to acinar proliferation. In the third month of foetal life, the Islets of Langerhans develop from the collecting tubules. At 12-18 weeks of age, islets were small and spherical. As the age of foetus increased, size and number of islets also increased. Islets were well encapsulated. In foetuses with age group 36-40 weeks, islets were larger and prominent and they were more concentrated towards the tail region as compared to the head and body. As noted by previous investigators, both the acinar and islet epithelia develop from the primitive pancreatic tubules which was noted by Conklin, Gupta. $\mathrm{V}$ et al, P. Robb and Achaya Anand. ${ }^{8,12-14}$

Human fetal pancreas and its cytogenesis was studied in detail by James L. Conklin. ${ }^{8}$ In his study the tissues from 54 human foetuses were subjected for Haematoxylin and Eosin method. Group wise arrangement and observations of all stages of development were similar to his findings.

During our investigation we obtained ducts (intra and interlobular) as compared with few acini and Islets in micro architecture of the foetal pancreas. (Dietrich Grube)..$^{15}$

Electron microscopic study of 12 fetal pancreas was done by Gupta Renu et al. At 12 weeks of gestation pancreatic acini were well formed and at 14weeks of gestation isolated endocrine cells in close proximity with the acini were seen. According to them it was suggested that both exocrine and endocrine part arise from common source i.e. from the ductal epithelium. This observation was also noted in our study.

Ferner and Stoeckenieus in 1950 studied 12 foetal pancreas and found islet buds by $10^{\text {th }}$ week of development and were divided into 3 stages of development. i.e. Single cell, Inselfield and Mantellinsel. He also recognised the alpha and beta cells in the human pancreatic islets as early as $10-13$ weeks by Gomori's method. ${ }^{10}$

In the current study, similar stages observed during 12-18 weeks, at 18-24 weeks and finally from 25 weeks onwards.
Hahnvon Dorsche $\mathrm{H}$ et al investigated 41 human pancreata from 14-26 weeks of development. He divided foetuses into 3 phases of development. Phase 1 (14-16 weeks) characterised by Islet buds which originated from the ducts. Phase 2 (17-20 weeks) showed islets were detached from the ducts and form mantle field islets. Phase 3 (21-26 weeks) showed islet cytology approximately similar to the adult human islets. The present work was correlated with the similar architecture. ${ }^{16}$

The development of both component exocrine as well as endocrine part of human fetal pancreas was studied by light microscopy. We were try to formulate normal different developmental stages of human fetal pancreas from 12 gestational weeks to full term. We observed similar morphological changes which coincides with previous studies.

The selection of an appropriate fetal pancreas during its developmental stage is of paramount importance for the successful transplantation of pancreas in patients of insulin dependent diabetes mellitus.

\section{Conclusion}

Human foetal pancreas at an optimal stage of its development is a suitable organ for transplantation in patients with insulin dependent diabetes mellitus. And the overview study of the acini and its growth help in pancreatic cancer. The success of pancreatic transplant requires the knowledge of development, morphology and islet genesis for cell replacement therapies.

\section{Acknowledgements}

All authors are thankful to the department of anatomy, KAMSRC\& KIMS, Hyderabad. Authors of this study also acknowledged to authors, editors \& publishers of all literature for this article has been reviewed and discussed.

\section{Conflict of Interest: Nil.}

\section{References}

1. Gupta Renu, Shukla Satyanarayan and Nayyar K Ashish. The histogenesis of developing foetal pancreas - an electron microscopic study. International Journal of Biomedical Research. 2014;5(11):695-699.

2. J.M.W. Slack. Developmental biology of the Pancreas. Development. 1995;121:1569-1580.

3. Asim Kumar Dutta. Essentials of Human Embryology. 5h edn. Kolkata: Current books international; 2005;143-144.

4. Bailey and love's Short Practice of Surgery. $24^{\text {th }}$ edn, Hodder education; 2004;1114.

5. Vincent. S. The relationship between the islets of Langerhans and the Zymogenous tissue of the pancreas. Lancet, 206:947-948.

6. Bencosme S.A. The histogenesis and cytology of the pancreatic islets in the rabbit. American journal of Anatomy. 1953;96:103-151.

7. Beard J. The Enzyme Treatment of Cancer. London: Chatto and Windus, 1911. 
8. Conklin J.A. Cytogenesis of human foetal pancreas. American journal of Anatomy. 1962;111:181-193.

9. Jones EA, Clement - Jones M, James OF, Wilson DI. $J$ Anat. 2001;198(P5):555- 9

10. Ferner, H, and W. Stoeckenius, Jr. Die Cytogenese des Insel systemsbeim Menschen. Ztschr. F. Zellforsch. u. Mikr. Anat, 1951;92:143-149.

11. E.C.CLAYDEN. Practical section cutting and staining. $3^{\text {rd }}$ edn. J. \& A. CHURCHILL Ltd; 1955;3-66.

12. Gupta, v, Garg, K., Raheja, S., Choudhry, R. and Tuli, A. The Histogenesis of Islets in the Human Foetal Pancreas. $J$ Ant Soc India. 2002;51(1):23-26.

13. Robb P. The Development of islets of Langerhans in the human foetus. Quarterly Journal of Experimental Physiology. 1961;46:335-343.

14. Achaya and Anand. Histogenesis of Pancreatic Islets in the human embryo. Journal of Anatomical society of India. 1966;(14)2:63-69.
15. Dietrich GRUBE and Reinhild BOHN. The Microanatomy of Human Islets of Langerhans, with Special Reference to Somatostatin (D-) Cells. Arch Histol Jap. 1983;46(3)327-353.

16. Hahn von Dorsche H, Reiher H, Hahn HJ. Phases in the early development of the human islet organ. Anat Anz.1988;166(1-5):69-76.

How to cite this article: Ramani T.V, Pratyusha M, Saritha S. Chronological study in microanatomy of human foetal pancreatic tissue. Indian J Clin Anat Physiol. 2018;5(4):559-563. 